\title{
Cross-Layer Optimized Multipath Routing for Video Communications in Wireless Networks
}

\author{
Sastry Kompella, Member, IEEE, Shiwen Mao, Member, IEEE, Y. Thomas Hou, Senior Member, IEEE, \\ and Hanif D. Sherali
}

\begin{abstract}
Traditionally, routing is considered solely as a network layer problem and has been decoupled from application layer objectives. Although such an approach offers simplicity in the design of the protocol stack, it does not offer good performance for certain applications such as video. In this paper, we explore the problem of how to perform routing with the objective of optimizing application layer performance. Specifically, we consider how to perform multipath routing for multiple description (MD) video in a multi-hop wireless network. We formulate this problem into an optimization problem with application performance metric as the objective function and routing and link layer considerations as constraints. We develop a formal branch-and-bound framework and exploit the so-called Reformulation-Linearization Technique (RLT) in the solution procedure. We show that this solution procedure is able to produce a set of routes whose objective value is within $(1-\epsilon)$ of the optimum. We use simulation results to substantiate the efficacy of the solution procedure and compare the performance with that under non-cross-layer approach.
\end{abstract}

Index Terms - Video communications, multipath routing, multiple description (MD) coding, optimization.

\section{INTRODUCTION}

$\mathbf{R}$ ECENTLY, there has been considerable interest in supporting video applications in multi-hop wireless networks (e.g., ad hoc networks or mesh networks). The dynamic characteristics associated with multi-hop wireless networks (e.g., mobility, topology change, radio fading and loss) have posed some unique challenges for video communications. Existing routing protocols for such networks (e.g., OLSR [5], DSR [8], AODV [14]) mainly focus on network layer connectivity problem and do not have explicit consideration for video application requirements. As a result, such single layer (network layer) approaches are not optimal to support video applications.

In this paper, we investigate the important problem of how to design routing protocols so as to optimally support

Manuscript received May 6, 2006; revised November 1, 2006. This research has been supported in part by the Office of Naval Research (ONR) under Grants N00014-03-1-0521 and N00014-05-1-0179, and by the National Science Foundation (NSF) under Grants DGE-9987586, CNS-0347390 and DMI-0552676. This work was completed while S. Kompella and S. Mao were with Virginia Tech, Blacksburg, VA.

S. Kompella is currently with Information Technology Division, U.S. Naval Research Laboratory, Washington, DC 20375 (e-mail: kompella@itd.nrl.navy.mil).

S. Mao is currently with the Department of Electrical and Computer Engineering, Auburn University, Auburn, AL 36849 (e-mail: smao@ieee.org).

Y.T. Hou is with the Bradley Department of Electrical and Computer Engineering, Virginia Tech, Blacksburg, VA 24061 (e-mail: thou@vt.edu).

H.D. Sherali is with the Grado Department of Industrial and Systems Engineering, Virginia Tech, Blacksburg, VA 24061 (e-mail: hanifs@vt.edu).

Digital Object Identifier 10.1109/JSAC.2007.070518. video applications in multi-hop wireless networks. We aim to develop a cross-layer approach where the routing decision takes explicit consideration of the application layer objective function. In other words, the routing engine at the network layer will not only find routes for connectivity, but will find optimal routes such that the application layer performance metric (e.g., PSNR) is maximized.

Throughout this investigation, we will employ multiple description (MD) video as our target application. MD video is an important coding technique for error resilience and control for multimedia applications [17] and has been recognized as an ideal candidate for video streaming in multi-hop wireless networks [10]. Under MD coding, multiple equivalent streams (or descriptions) are generated for a video source for transmission. At the receiver, any received subset of these descriptions can be combined to reconstruct the original video and the quality of the reconstructed video is commensurate with the number of received descriptions. This video coding technique is drastically different from traditional layered video coding, where video reconstruction hinges upon successful delivery of the base layer.

From cross-layer routing perspective, the problem is to find a set of routes (or paths) in multi-hop wireless networks, one for each video description such that the video distortion is minimized. The optimal multipath routing problem considered in this paper is formulated into a mixed-integer non-linear programming $(N L P)$ problem. Such problems are shown to be NP-hard in general [16]. In a previous work [9], we studied this problem and addressed it using Genetic Algorithms (GA). Although GA is an effective algorithm, it nevertheless is a metaheuristic which does not provide any performance bounds on how close the solution is to the optimal. As a result, a theoretical result for multipath routing for MD video remains an open problem.

In this paper, we aim to bridge this important theoretical gap in cross-layer optimization for video communications. We present a formal solution procedure based on the so-called branch-and-bound [12] framework, which aims to produce an $(1-\epsilon)$-optimal solution. Here $\epsilon>0$ is an arbitrarily small number reflecting required accuracy. A key component in the solution procedure is a novel method called the ReformulationLinearization Technique (RLT) [15], which in essence produces tight lower bound for a minimization problem. A global optimal solution can be obtained by embedding RLT into the branch-and-bound framework. That is, during each branch-and-bound iteration, we employ RLT to generate an LP relaxation for the corresponding sub-problem, and by solving 
this LP relaxation we generate a lower bound for the subproblem. The corresponding upper bound is computed by a suitable local search algorithm on the solution provided by the LP relaxation. Based on the bounds of all the sub-problems currently in the branch-and-bound tree, the gap between the lower and upper bounds of the original problem is narrowed iteratively, until they are within $\epsilon$ of each other.

The remainder of this paper is organized as follows. In Section II, we present the problem formulation. In Section III, we describe an RLT-based approach to reformulate and linearize the problem. Section IV presents a branch-and-boundbased solution procedure. In Section V, we briefly discuss implementation issues. Simulation results are presented in Section VI, and related work is discussed in Section VII. Section VIII concludes this paper.

\section{PROBLEM Formulation}

\section{A. Network Model}

We model a multi-hop wireless network as a directed graph $\mathcal{G}\{\mathcal{V}, \mathcal{E}\}$, where $\mathcal{V}$ is the set of vertexes representing wireless nodes and $\mathcal{E}$ the set of edges representing wireless links. We assume that nodes are reliable during the video session, but links may be up or down with certain probabilities. For our routing problem, we focus on network layer statistics, assuming that the physical and MAC layer dynamics from the underlying radio environment are reflected in these metrics. We characterize a link $\{i, j\} \in \mathcal{E}$ with (i) $b_{i j}$ : the available bandwidth of link $\{i, j\}$; (ii) $p_{i j}$ : the probability that link $\{i, j\}$ is "up"; (iii) $l_{i j}$ : average burst length for packet losses on link $\{i, j\}$. Based on these basic metrics, we can derive pathlevel bandwidth and failure probability, which are useful to characterize end-to-end performance at the video application layer (i.e., distortion). Table I summarizes the notation used in this paper.

\section{B. Video Distortion and Path Level Statistics}

1) Video Distortion: Consider a video session from video server $s$ to client $t$. We assume that the video is encoded into two descriptions, i.e., double description (DD) video, each with a rate $R_{h}$ bits/pixel, $h=1,2$. We consider DD video since it is most widely used for MD video [2]-[4], [10]. Let $d_{h}$ be the achieved distortion when only description $h$ is received, $h=1,2$, and $d_{0}$ the distortion when both descriptions are received. In this paper, we employ the following distortionrate function [1], [9]:

$$
\left\{\begin{array}{l}
d_{0}=\frac{2^{-2\left(R_{1}+R_{2}\right)}}{2^{-2 R_{1}}+2^{-2 R_{2}-2^{-2\left(R_{1}+R_{2}\right)}} \cdot \sigma^{2}} \\
d_{1}=2^{-2 R_{1}} \cdot \sigma^{2} \\
d_{2}=2^{-2 R_{2}} \cdot \sigma^{2}
\end{array}\right.
$$

where $\sigma^{2}$ is the variance of the source.

From end-to-end perspective, let $\pi_{00}$ denote the probability of receiving both descriptions, $\pi_{01}$ the probability of receiving description 1 only, $\pi_{10}$ the probability of receiving description 2 only, and $\pi_{11}$ the probability of losing both descriptions. Then, the expected average video distortion at the receiver can be approximated as:

$$
D=\pi_{00} \cdot d_{0}+\pi_{01} \cdot d_{1}+\pi_{10} \cdot d_{2}+\pi_{11} \cdot \sigma^{2} .
$$

TABLE I

NOTATION

\begin{tabular}{ll}
\hline Symbol & Definition \\
\hline $\mathcal{G}\{\mathcal{V}, \mathcal{E}\}$ & Graph representation of the network \\
$\mathcal{V}$ & Set of vertexes \\
$\mathcal{E}$ & Set of edges \\
$s$ & Source node \\
$t$ & Destination node \\
$\mathcal{P}$ & A path from $s$ to $t$ \\
$\{i, j\}$ & A link from node $i$ to node $j$ \\
$b_{i j}$ & Bandwidth of link $\{i, j\}$ \\
$p_{i j}$ & Success probability of link $\{i, j\}$ \\
$l_{i j}$ & Average length of loss burst on link $\{i, j\}$ \\
$R_{h}$ & Rate of description $h$ in bits/pixel, $h=1,2$ \\
$R$ & For balanced descriptions, $R=R_{1}=R_{2}$ \\
$d_{0}$ & Distortion when both descriptions are received \\
$d_{h}$ & Distortion when only description $h$ is received, \\
& $h=1,2$ \\
$T_{\text {on }}$ & Average distortion \\
$\Lambda$ & Average "up" period of the joint links \\
$\Psi$ & "up" to "down" transition prob. for $\mathcal{J}\left(\mathcal{P}_{1}, \mathcal{P}_{2}\right)$ \\
$\pi_{00}$ & "down" to "up" transition prob. for $\mathcal{J}\left(\mathcal{P}_{1}, \mathcal{P}_{2}\right)$ \\
$\pi_{01}$ & Probability of receiving both descriptions \\
$\pi_{10}$ & Probability of receiving description 1 only \\
$\pi_{11}$ & Probability of receiving description 2 only \\
$I_{i j}^{(h)}$ & Probability of losing both descriptions \\
$\alpha_{i j}$ & Routing index variables, defined in $(3)$ \\
$\beta_{i j}$ & "up" to "down" transition prob. of link $\{i, j\}$ \\
$p_{j n t}$ & Average success prob. of joint links \\
$p_{d j}(h)$ & Average success prob. of disjoint links on $\mathcal{P}_{h}$ \\
\hline &
\end{tabular}

Note that our solution procedure presented later in this paper does not depend on the specific structure of the distortion-rate function.

2) Path-Level Statistics: To characterize a path $\mathcal{P}_{h}$ between source node $s$ and destination node $t$, we define:

$$
I_{i j}^{(h)}= \begin{cases}1, & \text { if link }\{i, j\} \in \mathcal{P}_{h}, \\ 0, & \text { otherwise }\end{cases}
$$

An arbitrary path $\mathcal{P}_{h}$ can then be represented by a vector $\mathbf{I}^{(h)}$ of $|\mathcal{E}|$ elements, each corresponding to a link and having a binary value.

For a source-destination pair $\{s, t\}$, consider two given paths $\left[\mathcal{P}_{1}, \mathcal{P}_{2}\right]$ in $\mathcal{G}\{\mathcal{V}, \mathcal{E}\}$. Since we do not mandate "disjointedness" between the two paths, $\mathcal{P}_{1}$ and $\mathcal{P}_{2}$ may share nodes and links. For each link $\{i, j\}$, the aggregate description rate should be bounded by its available bandwidth as

$$
I_{i j}^{(1)} \cdot R_{1}+I_{i j}^{(2)} \cdot R_{2} \leq \rho \cdot b_{i j},
$$

where $\rho$ is a constant. For a video with coding rate $f$ frames/s and a resolution of $W \times V$ pixels/frame, we have $\rho=1 /(\kappa$. $W \cdot V \cdot f)$, where $\kappa$ is a constant determined by the chroma sub-sampling scheme (e.g, $\kappa=1.5$ for QCIF).

We now focus on how to compute the end-to-end path statistics. Similar to the approach in [2], [3], we classify the links into three sets: set one consisting of links shared by both paths, denoted as $\mathcal{J}\left(\mathcal{P}_{1}, \mathcal{P}_{2}\right)$, and the other two sets consisting of disjoint links on the two paths, denoted as $\overline{\mathcal{J}}\left(\mathcal{P}_{h}\right), h=1,2$, respectively. For disjoint portion of the paths, it suffices to model the packet loss as a Bernoulli event, since losses of the two descriptions are assumed to be independent on disjoint 


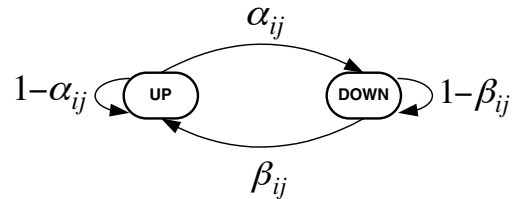

(a) A two-state link model

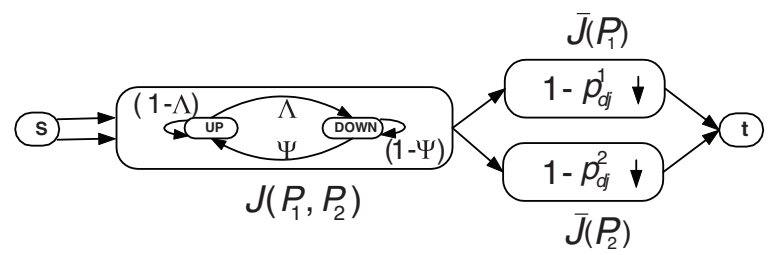

(b) A path model characterizing joint and disjoint links

Fig. 1. Link and path models.

portions. The success probabilities on the disjoint portions are:

$p_{d j}^{(h)}= \begin{cases}\prod_{\{i, j\} \in \overline{\mathcal{J}}\left(\mathcal{P}_{h}\right)} p_{i j}, & \text { if } \overline{\mathcal{J}}\left(\mathcal{P}_{h}\right) \neq \emptyset, h=1,2 \\ 1, & \text { otherwise, } h=1,2 .\end{cases}$

On the joint portion of the paths, losses on the two streams are correlated. In order to model such correlation, we model each shared link $\{i, j\}$ as an on-off process modulated by a discrete-time Markov chain, as shown in Figure 1(a). There is no packet loss when the link is "up"; all packets are dropped when the link is "down". Transition probabilities, $\left\{\alpha_{i j}, \beta_{i j}\right\}$, can be computed from the link statistics as $\beta_{i j}=1 / l_{i j}$ and $\alpha_{i j}=\left(1-p_{i j}\right) /\left(p_{i j} l_{i j}\right)$.

If there are $K$ shared links, the aggregate failure process of these links is a Markov process with $2^{K}$ states. In order to simplify the computation, we model the aggregate process as an on-off process. Specifically, we lump up all the states with at least one link failure into a single "down" state, while using the remaining state where all the links are in good condition as the "up" state. Let $T_{o n}$ be the average length of the "up" period. We have

$$
T_{\text {on }}=\frac{1}{1-\prod_{\{i, j\} \in \mathcal{J}\left(\mathcal{P}_{1}, \mathcal{P}_{2}\right)}\left(1-\alpha_{i j}\right)} .
$$

The transition probabilities of the aggregate on-off process can be computed as

$$
\Lambda=\frac{1}{T_{o n}}, \quad \Psi=\frac{p_{j n t}}{T_{o n}\left(1-p_{j n t}\right)},
$$

where $p_{j n t}$ is the average success probability of the joint portion, and

$$
p_{\text {jnt }}= \begin{cases}\prod_{\{i, j\} \in \mathcal{J}\left(\mathcal{P}_{1}, \mathcal{P}_{2}\right)} p_{i j}, & \text { if } \mathcal{J}\left(\mathcal{P}_{1}, \mathcal{P}_{2}\right) \neq \emptyset \\ 1, & \text { otherwise. }\end{cases}
$$

Note that $\Lambda=0$ and $\Psi=0$ if $\mathcal{J}\left(\mathcal{P}_{1}, \mathcal{P}_{2}\right)=\emptyset$.

The consolidated path model is illustrated in Figure 1(b), where $\mathcal{J}\left(\mathcal{P}_{1}, \mathcal{P}_{2}\right)$ is modeled as a two-state Markov process with parameters $\{\Lambda, \Psi\}$, and $\overline{\mathcal{J}}\left(\mathcal{P}_{h}\right)$ is modeled as a Bernoulli process with parameter $\left(1-p_{d j}^{(h)}\right), h=1,2$. With the consolidated path model, the joint probabilities of receiving the descriptions are:

$$
\left\{\begin{array}{l}
\pi_{00}=p_{j n t} \cdot(1-\Lambda) \cdot p_{d j}^{1} \cdot p_{d j}^{2} \\
\pi_{01}=p_{j n t} \cdot p_{d j}^{1} \cdot\left[1-(1-\Lambda) \cdot p_{d j}^{2}\right] \\
\pi_{10}=p_{j n t} \cdot\left[1-(1-\Lambda) p_{d j}^{1}\right] \cdot p_{d j}^{2} \\
\pi_{11}=1-p_{j n t} \cdot\left[p_{d j}^{1}+p_{d j}^{2}-(1-\Lambda) \cdot p_{d j}^{1} \cdot p_{d j}^{2}\right] .
\end{array}\right.
$$

\section{Problem Formulation}

We can now formulate the problem of multipath routing for MD video into the following mathematical programming problem.

\section{OPT-MR}

Minimize:

$$
D=\pi_{00} \cdot d_{0}+\pi_{01} \cdot d_{1}+\pi_{10} \cdot d_{2}+\pi_{11} \cdot \sigma^{2}
$$

\section{subject to:}

$$
\begin{aligned}
& \sum_{j \in \mathcal{V}} I_{i j}^{(h)}-\sum_{j \in \mathcal{V}} I_{j i}^{(h)} \\
& =\left\{\begin{array}{rll}
1, & \text { if } i=s, & i \in \mathcal{V}, h=1,2 \\
-1, & \text { if } i=t, & i \in \mathcal{V}, h=1,2 \\
0, & \text { otherwise, } &
\end{array}\right.
\end{aligned}
$$

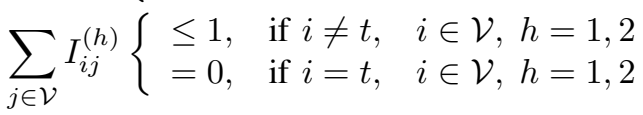

$$
\begin{aligned}
& I_{i j}^{(1)} \cdot R_{1}+I_{i j}^{(2)} \cdot R_{2} \leq \rho \cdot b_{i j}, \quad\{i, j\} \in \mathcal{E} \\
& I_{i j}^{(h)} \in\{0,1\}, \quad\{i, j\} \in \mathcal{E}, h=1,2 .
\end{aligned}
$$

In Problem OPT-MR, $\left\{I_{i j}^{(h)}\right\}$ are binary optimization variables (incorporated in $\pi_{00}, \pi_{01}, \pi_{10}$, and $\pi_{11}$ ). Constraint (11) guarantees that the paths originate at the source $s$ and terminate at the destination $t$, and constraint (12) ensures that the paths are loop-free. Constraint (13) guarantees that the links are stable. For a given pair of paths, the average video distortion $D$ is determined by the end-to-end statistics and the correlation of the paths, as given in (1) and (9).

The objective function (10) is a complex ratio of high-order exponentials of the $I$-variables. The objective evaluation of a pair of paths involves identifying the joint and disjoint portions, which is only possible when both paths are completely determined. Since such problems are NP-hard in general [7], and Problem OPT-MR does not appear to posses any special simplifying structure, it is likely to be NP-hard, although a formal proof is not given in this paper.

\section{REFORMULATION AND LINEARIZATION}

Our solution approach to problem OPT-MR is to embed the novel reformulation-linearization technique (RLT) in a branchand-bound framework [15]. RLT is a relaxation technique that can be used to produce tight polyhedral outer approximations or linear programming relaxations for an underlying nonlinear, non-convex polynomial programming problem. In the following, we first reformulate problem OPT-MR into a mixedinteger polynomial programming problem P-MR. Then, we replace all the non-linear terms and add the corresponding RLT constraints into the problem formulation, so as to obtain a linear programming relaxation of problem OPT-MR, denoted 


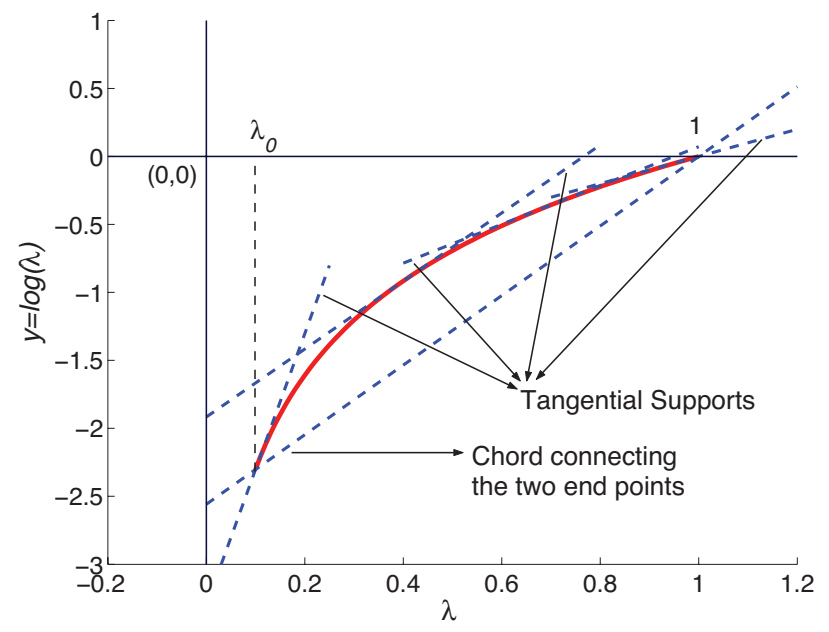

Fig. 2. Polyhedral outer approximation for $y=\log (\lambda)$ with $0<\lambda_{0} \leq$ $\lambda \leq 1$.

as L-MR. In the next section, we will develop a branch-andbound based procedure that finds $(1-\epsilon)$-optimal solution.

\section{A. Reformulating Problem OPT-MR}

As discussed, the objective function of problem OPT-MR is a complex function of exponential terms of the $I$-variables. Our first goal is to reformulate these terms, which will simplify the objective function and the constraints. Without loss of generality, we set $\sigma^{2}=1$ to simplify notation. Note that $\sigma^{2}$ only affects the absolute value of distortion, but not optimal routing selection.

In (9), there are four high order terms that need to be reformulated, namely, $p_{j n t}, p_{d j}^{(1)}, p_{d j}^{(2)}$, and $\Lambda$. From their definitions in (5) and (8), we can rewrite the success probabilities as:

$$
\left\{\begin{array}{l}
p_{j n t}=\prod_{\{i, j\} \in \mathcal{E}} p_{i j}^{\left\{I_{i j}^{(1)} \cdot I_{i j}^{(2)}\right\}} \\
p_{d j}^{(1)}=\prod_{\{i, j\} \in \mathcal{E}} p_{i j}^{\left\{I_{i j}^{(1)} \cdot\left(1-I_{i j}^{(2)}\right)\right\}} \\
p_{d j}^{(2)}=\prod_{\{i, j\} \in \mathcal{E}} p_{i j}^{\left\{I_{i j}^{(2)} \cdot\left(1-I_{i j}^{(1)}\right)\right\}}
\end{array}\right.
$$

Taking logarithms on both sides, we can convert the high order terms on the right-hand-side (RHS) of (15) into summations of quadratic terms of the $I$-variables, i.e.,

$$
\left\{\begin{array}{l}
\log \left(p_{j n t}\right)=\sum_{\{i, j\} \in \mathcal{E}}\left[I_{i j}^{(1)} \cdot I_{i j}^{(2)} \cdot \log \left(p_{i j}\right)\right] \\
\log \left(p_{d j}^{(1)}\right)=\sum_{\{i, j\} \in \mathcal{E}}\left[I_{i j}^{(1)} \cdot\left(1-I_{i j}^{(2)}\right) \cdot \log \left(p_{i j}\right)\right] \\
\log \left(p_{d j}^{(2)}\right)=\sum_{\{i, j\} \in \mathcal{E}}\left[I_{i j}^{(2)} \cdot\left(1-I_{i j}^{(1)}\right) \cdot \log \left(p_{i j}\right)\right] .
\end{array}\right.
$$

Similarly, we can rewrite $\Lambda$ according to (6) and (7) as

$$
\Lambda=1-\prod_{\{i, j\} \in \mathcal{E}}\left[1-\frac{1-p_{i j}}{p_{i j} \cdot l_{i j}}\right]^{\left\{I_{i j}^{(1)} \cdot I_{i j}^{(2)}\right\}} \text {. }
$$

Letting $\phi=1-\Lambda$ and taking logarithms on both sides, we have

$$
\log (\phi)=\sum_{\{i, j\} \in \mathcal{E}}\left[I_{i j}^{(1)} \cdot I_{i j}^{(2)} \cdot \log \left(h_{i j}\right)\right],
$$

where $h_{i j}=1-\frac{1-p_{i j}}{p_{i j} \cdot l_{i j}}$ is a constant for all $\{i, j\} \in \mathcal{E}$.
Having simplified the high-order terms, we now deal with the resulting constraints of the form $y=\log (\lambda)$, as shown in (16) and (18). We can linearize this logarithmic relationship over some tightly-bounded interval using a polyhedral outer approximation comprised of a convex envelope in concert with several tangential supports. For instance, if $\lambda$ is bounded as $0<\lambda_{0} \leq \lambda \leq 1$, these constraints can be written as follows.

$$
\left\{\begin{array}{l}
y \geq \frac{\log \left(\lambda_{0}\right)}{1-\lambda_{0}} \cdot(1-\lambda) \\
y \leq \log \left(\lambda_{k}\right)+\frac{\lambda-\lambda_{k}}{\lambda_{k}}, \quad k=1, \ldots, k_{\max }
\end{array}\right.
$$

where $\lambda_{k}=\lambda_{0}+\left(1-\lambda_{0}\right) \cdot(k-1) /\left(k_{\max }-1\right)$, for $k=$ $1,2, \cdots, k_{\max }$. A four-point tangential approximation can be obtained by letting $k_{\max }=4$, as illustrated in Figure 2 . The corresponding convex envelope consists of a chord connecting the two end points, which is used in combination with tangential supports at four points including the two end points. As a result, every logarithmic relationship specified in (16) and (18) translates to five linear constraints as described in (19). Note that such polyhedral outer approximations will be iteratively tightened during the branch-and-bound procedure (see Section IV).

Substituting $\phi$, we can rewrite constraint (9) as

$$
\left\{\begin{array}{l}
\pi_{00}=p_{j n t} \cdot p_{d j}^{(1)} \cdot p_{d j}^{(2)} \cdot \phi \\
\pi_{01}+\pi_{00}=p_{j n t} \cdot p_{d j}^{(1)} \\
\pi_{10}+\pi_{00}=p_{j n t} \cdot p_{d j}^{(2)} \\
\pi_{00}+\pi_{01}+\pi_{10}+\pi_{11}=1
\end{array}\right.
$$

and the objective function as

$$
\begin{array}{r}
D=\pi_{00} \cdot d_{0}+\left(p_{j n t} \cdot p_{d j}^{(1)}-\pi_{00}\right) \cdot d_{1}+\left(p_{j n t} \cdot p_{d j}^{(2)}-\right. \\
\left.\pi_{00}\right) \cdot d_{2}+\left(1+\pi_{00}-p_{j n t} \cdot p_{d j}^{(1)}-p_{j n t} \cdot p_{d j}^{(2)}\right) .
\end{array}
$$

This reduces OPT-MR into a mixed-integer polynomial programming problem $\mathrm{P}-\mathrm{MR}$, with the objective being minimizing $D$ in (21). The constraints of P-MR include the original constraints (11)-(14), the reformulated constraint (20), and the new constraints derived from reformulating the logarithmic terms (16) and (18) [in the form of (19)].

\section{B. Linearizing Problem P-MR}

Although greatly simplified, problem P-MR is still a polynomial programming problem, which is NP-hard in general [15]. In this section, we linearize problem P-MR by employing RLT, which involves variable substitutions and introducing linear RLT bound-factor constraints.

Consider a quadratic product term of the form $\left(p_{d j}^{(1)} \cdot p_{d j}^{(2)}\right)$. By introducing a new variable $z_{0}=p_{d j}^{(1)} \cdot p_{d j}^{(2)}$, we can substitute the $\left(p_{d j}^{(1)} \cdot p_{d j}^{(2)}\right)$ terms in (20) and (21) with $z_{0}$, thus removing this quadratic term from the objective function and constraints. Assuming $p_{d j}^{(1)}$ and $p_{d j}^{(2)}$ are each bounded as $\left(p_{d j}^{(1)}\right)_{L} \leq p_{d j}^{(1)} \leq\left(p_{d j}^{(1)}\right)_{U}$ and $\left(p_{d j}^{(2)}\right)_{L} \leq p_{d j}^{(2)} \leq\left(p_{d j}^{(2)}\right)_{U}$, respectively, we can add the following relational constraints, 
which are known as the RLT bound-factor product constraints:

$$
\left\{\begin{array}{l}
\left\{\left[p_{d j}^{(1)}-\left(p_{d j}^{(1)}\right)_{L}\right] \cdot\left[p_{d j}^{(2)}-\left(p_{d j}^{(2)}\right)_{L}\right]\right\}_{L S} \geq 0 \\
\left\{\left[p_{d j}^{(1)}-\left(p_{d j}^{(1)}\right)_{L}\right] \cdot\left[\left(p_{d j}^{(2)}\right)_{U}-p_{d j}^{(2)}\right]\right\}_{L S} \geq 0 \\
\left\{\left[\left(p_{d j}^{(1)}\right)_{U}-p_{d j}^{(1)}\right] \cdot\left[p_{d j}^{(2)}-\left(p_{d j}^{(2)}\right)_{L}\right]\right\}_{L S} \geq 0 \\
\left\{\left[\left(p_{d j}^{(1)}\right)_{U}-p_{d j}^{(1)}\right] \cdot\left[\left(p_{d j}^{(2)}\right)_{U}-p_{d j}^{(2)}\right]\right\}_{L S} \geq 0
\end{array}\right.
$$

where $\{\cdot\}_{L S}$ denotes a linearization step under the substitution $z_{0}=p_{d j}^{(1)} \cdot p_{d j}^{(2)}$. Expanding the above inequalities and substituting $z_{0}=p_{d j}^{(1)} \cdot p_{d j}^{(2)}$, we obtain the following RLT constraints for $z_{0}$.

$$
\left\{\begin{array}{l}
\left(p_{d j}^{(1)}\right)_{L} p_{d j}^{(2)}+\left(p_{d j}^{(2)}\right)_{L} p_{d j}^{(1)}-z_{0} \leq\left(p_{d j}^{(1)}\right)_{L}\left(p_{d j}^{(2)}\right)_{L} \\
\left(p_{d j}^{(1)}\right)_{L} p_{d j}^{(2)}+\left(p_{d j}^{(2)}\right)_{U} p_{d j}^{(1)}-z_{0} \geq\left(p_{d j}^{(1)}\right)_{L}\left(p_{d j}^{(2)}\right)_{U} \\
\left(p_{d j}^{(1)}\right)_{U} p_{d j}^{(2)}+\left(p_{d j}^{(2)}\right)_{L} p_{d j}^{(1)}-z_{0} \geq\left(p_{d j}^{(1)}\right)_{U}\left(p_{d j}^{(2)}\right)_{L} \\
\left(p_{d j}^{(1)}\right)_{U} p_{d j}^{(2)}+\left(p_{d j}^{(2)}\right)_{U} p_{d j}^{(1)}-z_{0} \leq\left(p_{d j}^{(1)}\right)_{U}\left(p_{d j}^{(2)}\right)_{U} .
\end{array} .\right.
$$

By adding the linear RLT bound-factor constraints for $z_{0}$ into the problem formulation, we can therefore replace the secondorder term $p_{d j}^{(1)} \cdot p_{d j}^{(2)}$ with the linear term $z_{0}$ in (20) and (21).

Similarly, we define new variables for all the remaining non-linear terms in the reformulated problem $\operatorname{OPT-MR}(p)$, including $z_{1}=p_{j n t} \cdot p_{d j}^{(1)}, z_{2}=p_{j n t} \cdot p_{d j}^{(2)}$, and $z_{3}=z_{0} \cdot \phi$, and make substitutions in the same manner. We can then rewrite the objective function (21) and constraints (20) as

$$
\begin{aligned}
D= & \pi_{00} \cdot d_{0}+\left(z_{1}-\pi_{00}\right) \cdot d_{1}+ \\
& \left(z_{2}-\pi_{00}\right) \cdot d_{2}\left(1+\pi_{00}-z_{1}-z_{2}\right)
\end{aligned}
$$

and

$$
\left\{\begin{array}{l}
\pi_{01}+\pi_{00}=z_{1} \\
\pi_{10}+\pi_{00}=z_{2} \\
\pi_{00}+\pi_{01}+\pi_{10}+\pi_{11}=1
\end{array}\right.
$$

The constraints derived from reformulating the logarithmic terms (16) and (18) [in the form of (19)] can also be linearized by substituting $z_{i j}=I_{i j}^{(1)} \cdot I_{i j}^{(2)}$, and by introducing the corresponding linear RLT bound-factor constraints, for all $\{i, j\} \in \mathcal{E}$.

As a result, we obtain a linear programming relaxation problem L-MR, which can be solved in polynomial-time.

\section{A Solution Procedure}

\section{A. Overview of the Branch-and-Bound Framework}

Branch-and-bound is an algorithmic method for solving optimization problems, especially in discrete and combinatorial optimization [15]. Under branch-and-bound, the original problem is first relaxed using a suitable relaxation technique to obtain an easier-to-solve, lower-bounding problem. In our approach, we used RLT to reformulate and linearize OPTMR into an LP relaxation L-MR. The optimal solution to this LP relaxation provides a lower bound $L B$ for the original problem. Since such an LP relaxation usually yields an infeasible solution to the original problem, a local search algorithm should be employed to obtain a feasible solution to the original problem. The resulting feasible solution then provides an upper bound $U B$ for the original problem.
Under branch-and-bound framework, the original problem $O$ (or OPT-MR) is partitioned into sub-problems, each having a smaller feasible solution space, based on the solution provided by the LP relaxation. New sub-problems are organized as a branch-and-bound tree, while this partitioning or branching process is carried out recursively to obtain two new sub-problems at each node of the tree.

The sub-problems are also inserted into a problem list $L$, which records the active nodes in the branch-and-bound tree structure. More specifically, in the beginning, the problem list $L$ is initialized with the original problem $O$. At any given iteration, the lower and upper bounds for $O$ are computed as

$$
\left\{\begin{array}{l}
L B=\min \left\{L B_{k}: \text { Problem } k \in L\right\} \\
U B=\min \left\{U B_{k}: \text { all nodes } k \text { explored thus far }\right\} .
\end{array}\right.
$$

The method proceeds by choosing the next problem to partition from the problem list. In our approach, the problem $k \in L$ having the smallest $L B_{k}$ is chosen. This problem $k$ is then partitioned into two sub-problems $k_{1}$ and $k_{2}$, which replace problem $k$ in $L$. Every time a problem $k$ is added to the list, $L B_{k}$ and $U B_{k}$ are computed, and the $L B$ and $U B$ for the original problem $O$ are updated. At any given iteration, if $L B \geq(1-\epsilon) \cdot U B$, the procedure terminates and we have an $(1-\epsilon)$-optimal solution. Also, for any problem $k$ in the problem list, if $L B_{k} \geq(1-\epsilon) \cdot U B$, no globally optimal solution that improves beyond the $\epsilon$-tolerance can exist in the sub-space of the feasible region represented by this node. Therefore, this node can be removed (or fathomed) from the branch-and-bound tree. In this manner, the branch-and-bound process can fathom certain branches or nodes of the tree, eliminating them from further exploration. The effectiveness of the branch-and-bound procedure depends strongly on that of the employed fathoming strategy.

As far as the partitioning process is concerned, the original feasible solution space $\Omega$ is decomposed into two corresponding hyper-rectangles, based on a so-called branching variable. In RLT, the discrepancy between an RLT substitution variable (e.g., $z_{0}$ in Section III-B) and the corresponding non-linear term that this variable represents (e.g., $p_{d j}^{(1)} \cdot p_{d j}^{(2)}$ ) is called the relaxation error. In our algorithm, the branching variable is chosen to be the one that yields the largest relaxation error. Such a branching rule ensures that all the discrepancies between the RLT substitution variables and the corresponding non-linear terms will be driven to zero as the algorithm evolves over iterations.

\section{B. Details of the Solution Procedure}

We now describe the details of the solution procedure, which we call ALG $(\epsilon)$. Figure 3 shows the flowchart of this algorithm. The iterative branch-and-bound algorithm terminates when either the lower bound for the original problem is within $(1-\epsilon)$ of the upper bound, i.e., $L B \geq(1-\epsilon) \cdot U B$, or the problem list $L$ is empty. The operation of each step in $\operatorname{ALG}(\epsilon)$ is described in the following.

1) Initialization and Relaxation: We start by initializing the current "best" solution, denoted as $\psi^{*}$, with the solution $\bar{\psi}$ obtained as described below, and the current "best" upper bound $U B$ as the objective value obtained using this solution 


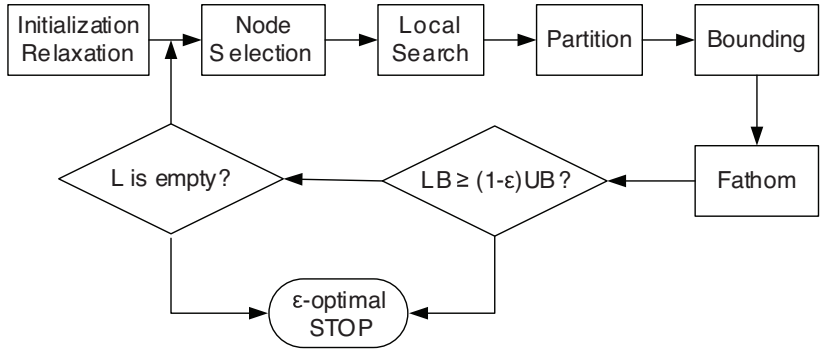

Fig. 3. Flowchart of the solution procedure.

$\bar{\psi}$. We first solve the relaxed problem L-MR to obtain a possibly infeasible solution $\hat{\psi}$, for the original problem. For example, due to the RLT relaxation, the binary $I$-variables in $\hat{\psi}$ could actually be fractional. If $\hat{\psi}$ is already feasible to problem OPT-MR, we set $\bar{\psi}=\hat{\psi}$ and then obtain a feasible solution in one iteration. Otherwise, we apply a local search algorithm (will be described in Section IV-B.3) to obtain a rounded feasible solution $\bar{\psi}$ to problem OPT-MR. If necessary, we can also perform a restricted search by making a limited perturbation around the rounded feasible solution $\bar{\psi}$ in order to obtain an even better solution. This can be achieved by incorporating a new constraint to the LP relaxation,

$$
\sum_{\{i, j\}: \bar{\psi}_{i j}^{(h)}=0} \psi_{i j}^{(h)}+\sum_{\{i, j\}: \bar{\psi}_{i j}^{(h)}=1}\left[1-\psi_{i j}^{(h)}\right] \leq r_{h},
$$

for some integral deviation tolerance $r_{h}, h=1,2$.

That is, a feasible solution $\bar{\psi}$ is obtained by solving the root node of the branch-and-bound tree and applying an efficient local search to the resulting solution. Due to the properly designed RLT relaxations (see Section III), the solution $\bar{\psi}$ is highly competitive in itself, and in many cases it achieves $(1-\epsilon)$-optimality.

Once $\psi^{*}$ is initialized, we then initialize the problem list $L$ with the original problem (denoted as $O$ ). We denote the objective value obtained from the LP relaxation as the lower bound $L B_{1}$ for Problem $O$. Also, since this is the only problem in the problem list, we initialize $L B_{1}$ as the current "best" lower bound $L B$ for the original problem, i.e., set $L B=L B_{1}$.

2) Node Selection: At every iteration, problem $k$ (or the corresponding node in the branch-and-bound tree) that has the minimum $L B_{k}$ among all the problems $k \in L$ is selected. As discussed before, this problem is indicative of the lower bound for the original problem. Subsequent operations of local search, partitioning and bounding are performed on this problem $k$.

3) Local Search: As discussed in Section IV-A, the solution to the relaxation problem $k$ that is selected in the node selection step, is usually infeasible to the original problem $O$. This is especially true if the original problem involves binary variables (i.e., the $I$-variables could be fractions). A local search algorithm should be used to find a feasible solution to the original problem starting from the infeasible lower bounding solution.

Let $\hat{\psi}$ be the infeasible (or fractional) solution obtained by solving the LP relaxation of the original problem. Starting from this fractional solution, we may solve the following problem to obtain a feasible path for $h=1,2$ respectively:

$$
\text { Minimize } \sum_{\{i, j\} \in \mathcal{E}}\left[-\hat{I}_{i j}^{(h)}\right] \cdot I_{i j}^{(h)}
$$

subject to the flow constraints, as described in (11) and (12). Note that for an optimization variable $y, \hat{y}$ denotes its value in the infeasible solution $\hat{\psi}$. Solving these shortest path problems provides us with a rounded heuristic solution $\bar{\psi}$ that has a tendency to round up relatively higher-valued components of $\hat{\psi}$ and round down relatively lower-valued components. The distortion value of the rounded solution $\bar{\psi}$ is an upper bound for this subproblem, i.e., $U B_{k}$.

4) Partitioning : The objective of the partitioning step is to find the branching variable that will enable us to split the feasible solution space $\Omega_{k}$ of problem $k$ into two solution sub-spaces $\Omega_{k_{1}}$ and $\Omega_{k_{2}}$. In $\operatorname{ALG}(\epsilon)$, we need to consider three classes of optimization variables for partitioning, i.e., the binary $I$-variables, the substitution variables (e.g., $z_{0}$ ), and the logarithm substitution terms [e.g., $\phi$ in (18)].

When partitioning based on the $I$-variables, we need to select a variable that will offer the highest gain in terms of improving the objective value. For this purpose, we should choose the $I$-variable that is fractional and closest to 0.5 . A strategy that works well is to first find the index variable pair $\left\{I_{i j}^{(1)}, I_{i j}^{(2)}\right\}$, for all $\{i, j\} \in \mathcal{E}$ that gives the largest discrepancy between the RLT substitution variable $\hat{z}_{i j}$ and the corresponding non-linear product $\left(\hat{I}_{i j}^{(1)} \cdot \hat{I}_{i j}^{(2)}\right)$ (see Section IIIB). We then choose $I_{i j}^{(1)}$ or $I_{i j}^{(2)}$ to partition the problem (by fixing it to 0 or 1) depending on which variable is closer to 0.5 . We break ties arbitrarily.

In addition to the $I$-variables, we also need to examine branching decisions based on the substitution variables such as $z_{0}=p_{d j}^{(1)} \cdot p_{d j}^{(2)}$. For such variables, we first find the maximum relaxation error between the substitution variable and the corresponding product term, say, $\left|\hat{p}_{d j}^{(1)} \cdot \hat{p}_{d j}^{(2)}-\hat{z}_{0}\right|$. We then verify whether the following condition is satisfied:

$$
\begin{aligned}
& {\left[\left(p_{d j}^{(1)}\right)_{U}-\left(p_{d j}^{(1)}\right)_{L}\right] \min \left[\hat{p}_{d j}^{(1)}-\left(p_{d j}^{(1)}\right)_{L},\left(p_{d j}^{(1)}\right)_{U}-\hat{p}_{d j}^{(1)}\right] \geq} \\
& {\left[\left(p_{d j}^{(2)}\right)_{U}-\left(p_{d j}^{(2)}\right)_{L}\right] \min \left[\hat{p}_{d j}^{(2)}-\left(p_{d j}^{(2)}\right)_{L},\left(p_{d j}^{(2)}\right)_{U}-\hat{p}_{d j}^{(2)}\right] .}
\end{aligned}
$$

If this condition holds true, we partition the solution space $\Omega_{k}$ of problem $k$ into two new regions $\Omega_{k_{1}}$ and $\Omega_{k_{2}}$, by dividing the range $\left[\left(p_{d j}^{(1)}\right)_{L},\left(p_{d j}^{(1)}\right)_{U}\right]$ into two subregions $\left[\left(p_{d j}^{(1)}\right)_{L}, \hat{p}_{d j}^{(1)}\right]$ and $\left(\hat{p}_{d j}^{(1)},\left(p_{d j}^{(1)}\right)_{U}\right]$. Otherwise, we partition $\Omega_{k}$ by dividing $\left[\left(p_{d j}^{(2)}\right)_{L},\left(p_{d j}^{(2)}\right)_{U}\right]$ into $\left[\left(p_{d j}^{(2)}\right)_{L}, \hat{p}_{d j}^{(2)}\right]$ and $\left[\hat{p}_{d j}^{(2)},\left(p_{d j}^{(2)}\right)_{U}\right]$.

Finally, the branching decisions also include the logarithm substitution terms, e.g., $\phi$ in (18). In such cases, we first find the variable that gives the greatest discrepancy between the logarithm value, say, $\log (\hat{\phi})$ and the RHS of the corresponding substitution [e.g., (18)] among all such terms, and then either bisect the interval of this variable (e.g., $\left.\left[(\phi)_{L},(\phi)_{U}\right]\right)$ evenly, or divide this interval at the point $\hat{\phi}$.

5) Bounding : In the bounding step, we solve the RLT relaxation for the two sub-problems identified in the partitioning step, and obtain their corresponding lower bounds $L B_{k_{1}}$ 
TABLE II

Performance of the Proposed Algorithm $(\epsilon=0.01)$

\begin{tabular}{ccc}
\hline Number of Nodes & Mean Computation Time(sec) & Variance \\
\hline 20 & 0.074 & 0.002 \\
30 & 0.381 & 0.282 \\
50 & 0.858 & 0.460 \\
100 & 6.408 & 3.196 \\
\hline
\end{tabular}

and $L B_{k_{2}}$, thereby updating the incumbent lower bounding solution. The corresponding upper bounds, i.e., $U B_{k_{1}}$ and $U B_{k_{2}}$, are obtained by applying the local search algorithm starting from the relaxation solutions obtained, and the current $L B$ and $U B$ values for the original problem $O$ are updated according to (24). If any of the following conditions

$$
(1-\epsilon) \cdot U B>L B_{k_{1}} \text { and }(1-\epsilon) \cdot U B>L B_{k_{2}}
$$

are satisfied, we add the corresponding problem into the problem list $L$, and remove problem $k$ from the list.

6) Fathoming: For any problem $k$ in the problem list $L$, if $L B_{k} \geq(1-\epsilon) \cdot U B$, then the sub-space corresponding to this problem does not contain any solution that improves beyond the $\epsilon$-tolerance of the incumbent solution. Therefore, we can prune this problem from the problem list.

\section{IMPLEMENTATIONS CONSIDERATIONS}

We find that the solution procedure is suitable for implementation as a link-state algorithm, which requires network topology and link statistics databases at a node. For implementation, we propose to build our algorithm on top of the so-called proactive routing protocols, such as Optimized Link State Routing (OLSR) [5]. Due to its proactive nature, OLSR maintains an up-to-date global network topology information in its link-state database. Once such information is available, computation can be performed to find a set of paths, and source routing can be used for each video description.

\section{Simulation Studies}

Our simulation study consists of two parts. In the first part, we will examine the convergence behavior and complexity issue of the proposed algorithm. In the second part, we will demonstrate the performance advantage of the proposed crosslayer approach over a non-cross-layer approach.

Throughout our simulation study, we consider a multi-hop wireless network deployed over a rectangular region, where the connectivity between the nodes is determined by the radio transmission range. The size of the area depends on the network size (total number of nodes) and will be described when we introduce the specific network. The source node $s$ and destination node $t$ are chosen randomly from the nodes in the network.

At the link level, we associate each link with a failure probability, available bandwidth, and mean burst length (for packet loss). Specifically, the failure probability is taken uniformly between $[0.01,0.3]$; the available bandwidth is taken with equal probability from the set $[100,150,200,250,300,350,400] \mathrm{Kb} / \mathrm{s}$; and the mean burst length is chosen uniformly between $[2,6]$. For $\operatorname{ALG}(\epsilon)$, we set $\epsilon=0.01$ (or $1 \%$ ). We implement the BB/RLT solution
TABLE III

AVERAGE DISTORTION VALUES FOR DIFFERENT NETWORK SIZES

$$
(\epsilon=0.01)
$$

\begin{tabular}{ccc}
\hline Number of Nodes & \multicolumn{2}{c}{ Mean Distortion } \\
\cline { 2 - 3 } & ALG $(\epsilon)$ & 2-SP \\
\hline 20 & 0.515 & 0.589 \\
30 & 0.516 & 0.591 \\
50 & 0.508 & 0.635 \\
100 & 0.512 & 0.575 \\
\hline
\end{tabular}

procedure in C program and use the LINDO API 3.0 for solving the LP relaxation problem.

\section{A. Convergence Behavior of Solution Procedure}

We first examine the convergence behavior of the solution procedure for different network sizes and topologies. We consider 20-, 30-, 50-, and 100-node networks within a 300m x $300 \mathrm{~m}, 400 \mathrm{~m} \times 400 \mathrm{~m}, 500 \mathrm{~m} \times 500 \mathrm{~m}$, and $1000 \mathrm{~m} \times 1000 \mathrm{~m}$ rectangular regions, respectively. The transmission range for each node is assumed to be $150 \mathrm{~m}$. The description rates are $R_{1}=R_{2}=128 \mathrm{~Kb} / \mathrm{s}$. For each network size, we generate 100 topologies and run 100 computations to obtain the mean and variance of convergence time. Table II shows the convergence time performance for the networks with $\epsilon=0.01$. The $\operatorname{ALG}(\epsilon)$ algorithm was run on a standard desktop PC with a Pentium-4 $2.4 \mathrm{GHz}$ processor and $512 \mathrm{MB}$ memory. As shown in Table II, the computational time for convergence to $\epsilon=0.01$ is very fast for small to moderate sized network.

We notice that for most experiments, the algorithm converges very quickly to the $(1-\epsilon)$-optimal solutions. To see the iterative convergence behavior of the solution procedure, we intentionally pick an experiment with a convergence time longer than the average. Figures 4 and 5 show such instances for the 50-node network and 100-node network, respectively for description rates of $R_{1}=R_{2}=320 \mathrm{~Kb} / \mathrm{s}$. For this particular 50-node network, the gap between upper bound $U B$ and lower bound $L B$ converges to $\epsilon=1 \%$ (i.e., $L B \geq$ $99 \% \cdot U B$ ) after the 57 th iteration in about 1.87 s. Similarly, for the selected 100-node network, the solution procedure converges to $\epsilon=1 \%$ after the 39th iteration in about 9.26 s. Figures 4(b) and 5(b) show the final optimal paths obtained by $\operatorname{ALG}(\epsilon)$ for the 50- and 100-node networks respectively.

\section{B. Comparison with Non-Cross-Layer Routing}

In this section, we compare our cross-layer routing approach with a popular non-cross-layer approach. For the latter, we consider the $k$-shortest path (SP) routing algorithm [6], with $k=2$ or 2-SP for DD video. We use hop count as the routing metric in the 2-SP algorithm.

1) Distortion Comparison: Table III compares the mean distortion achieved using our ALG $(\epsilon)$ and 2-SP for 4 network sizes $(20,30,50$ and 100 nodes) with $\epsilon=0.01$ and $R=$ $320 \mathrm{~Kb} / \mathrm{s}$. Again, for each network size, we generate 100 topologies and run 100 computations to obtain the mean distortion. As shown in the second and third columns in Table III, the distortion values under our cross-layer solution are consistently smaller than those under the 2-SP (non-crosslayer) solution. 


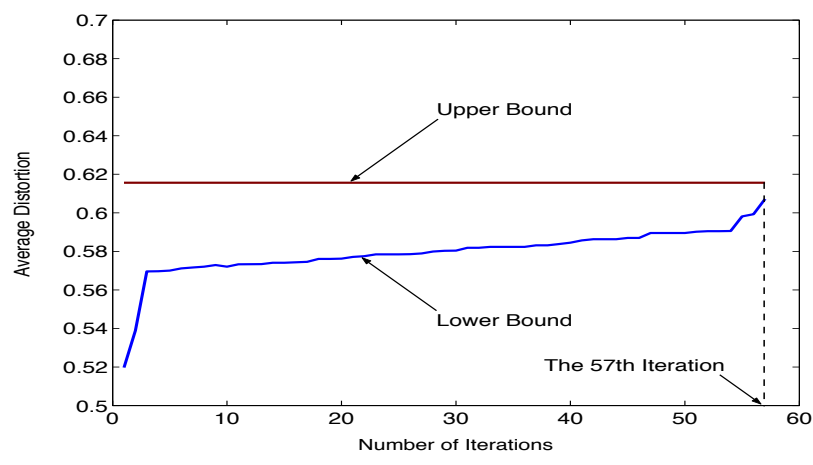

(a) Convergence behavior

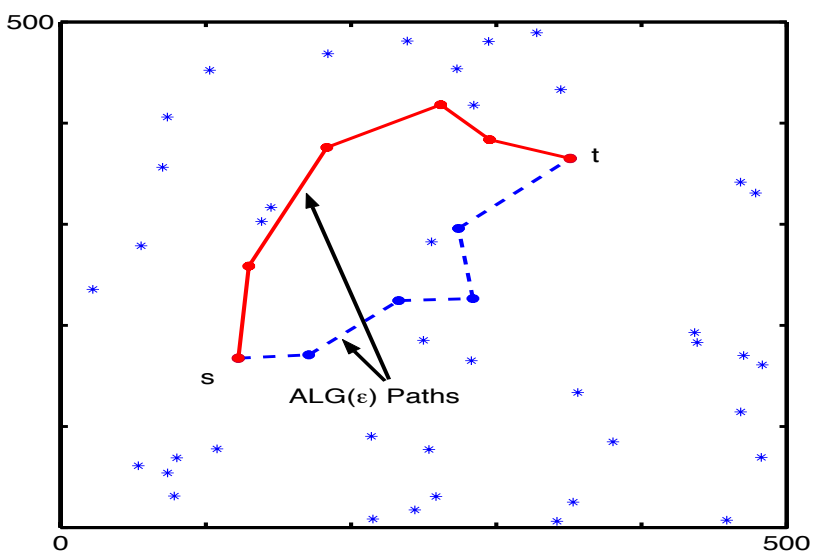

(b) $(1-\epsilon)$-optimal paths for DD video

Fig. 4. Convergence behavior and final optimal paths for a 50-node network.

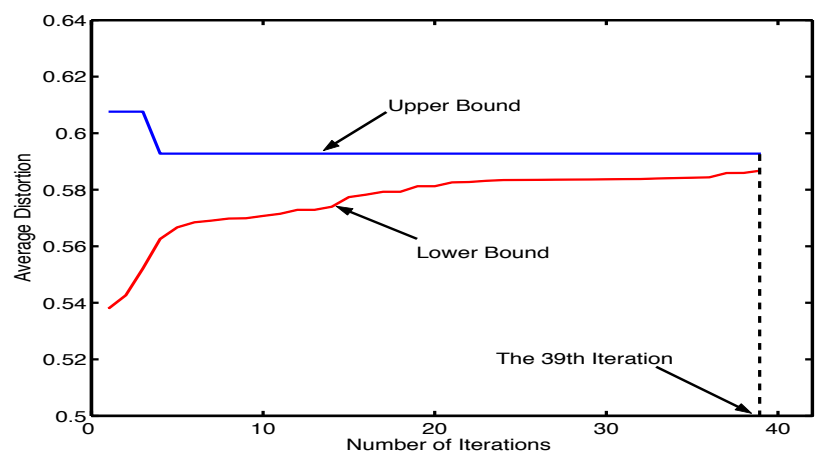

(a) Convergence behavior

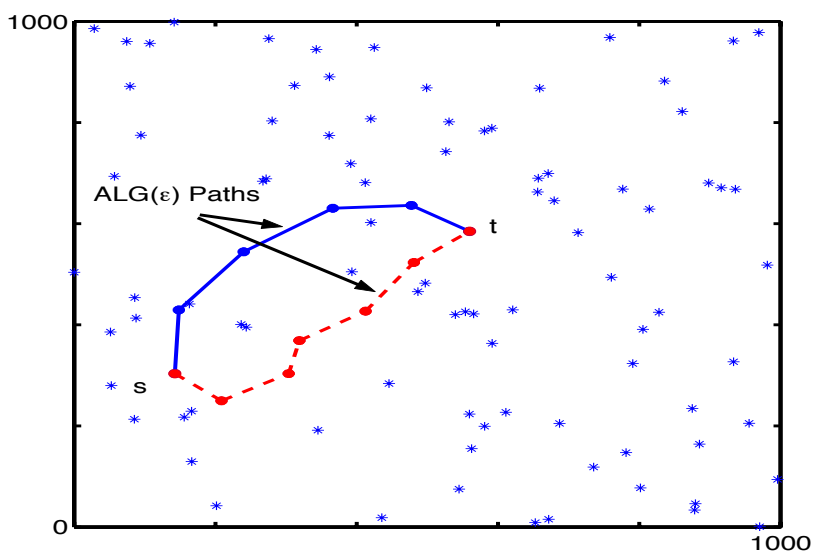

(b) $(1-\epsilon)$-optimal paths for DD video

Fig. 5. Convergence behavior and final optimal paths for a 100-node network.
TABLE IV

AVERAGE PSNR VALUES (DB) FOR DIFFERENT NETWORK SIZES

$$
(\epsilon=0.01)
$$

\begin{tabular}{ccc}
\hline Number of Nodes & \multicolumn{2}{c}{ Average PSNR } \\
\cline { 2 - 3 } & ALG $(\epsilon)$ & 2-SP \\
\hline 20 & 28.735 & 19.656 \\
30 & 27.313 & 17.203 \\
50 & 31.865 & 17.033 \\
100 & 29.809 & 20.418 \\
\hline
\end{tabular}

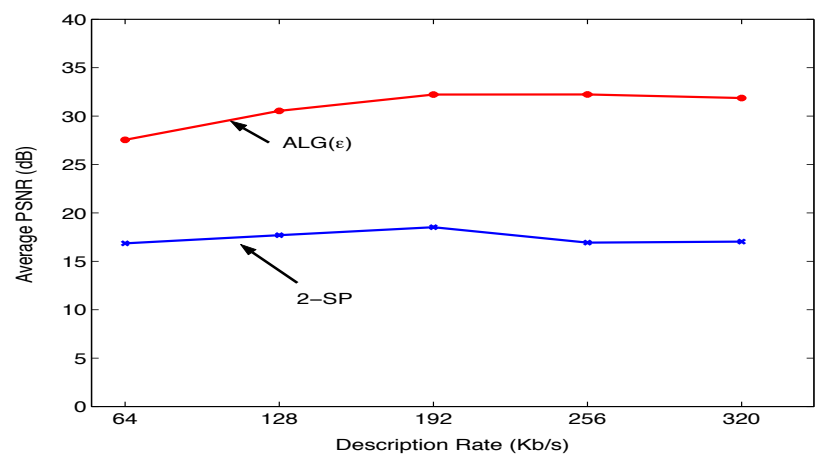

Fig. 6. Average PSNR values for different description rates.

2) Video Quality Comparison: We now encode a video sequence in order to transmit the DD video over the network, and compare the video quality at the receiver (measured using PSNR) under our cross-layer approach and the 2-SP approach. There are many ways to generate MD video (see [17]). We chose a time-domain partitioning coding scheme, where two descriptions are generated by separating the evenand odd-numbered frames and encoding them separately. This simple time-domain partitioning method is widely used in video streaming studies [2]-[4], [10]. An H.263+ like codec is implemented to generate the two descriptions. This codec encodes the video sequence into two balanced descriptions (i.e., $R_{1}=R_{2}$ ). The QCIF sequence "Foreman" (400 frames) is encoded at $15 \mathrm{fps}$ for each description. A $10 \%$ macroblock level intra-refreshment is used. Each Group of Blocks (GOB) is carried in a different packet. When a GOB is corrupted, the decoder applies a simple error concealment scheme by copying the corresponding slice from the most recent, correctly received frame.

The second and third columns in Table IV lists the average PSNR performance (in $\mathrm{dB}$ ) achieved using $\operatorname{ALG}(\epsilon)$ and 2SP for 4 network sizes $(20,30,50$ and 100 nodes) with $\epsilon=0.01$ and $R=320 \mathrm{~Kb} / \mathrm{s}$. We find that the average PSNR values obtained using $\operatorname{ALG}(\epsilon)$ are much higher than those obtained using 2-SP algorithm. This is consistent with the results obtained for distortion comparison shown earlier.

We now vary the rate of each video description from 64 $\mathrm{Kb} / \mathrm{s}$ to $320 \mathrm{~Kb} / \mathrm{s}$ for the 50 -node network and compare the PSNR performance under our solution and 2-SP. This results are shown in Figure 6. Note that as the description rate $R$ increases, more links will become ineligible during path selection process. Again, we find that our cross-layer approach provides higher PSNR over the 2-SP approach under all description rates.

To illustrate the quality of video frames, we plot a sample video frame from the the original video in Figure 7(a) and 


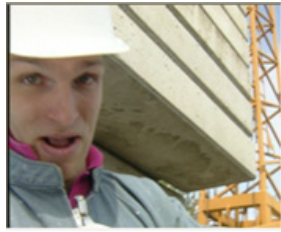

(a) Original

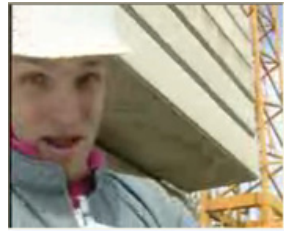

(b) $128 \mathrm{~Kb} / \mathrm{s}, \operatorname{ALG}(\epsilon)$

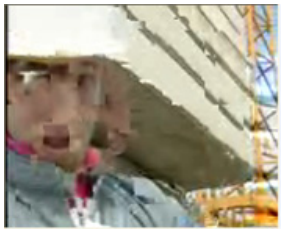

(c) $128 \mathrm{~Kb} / \mathrm{s}, 2-\mathrm{SP}$

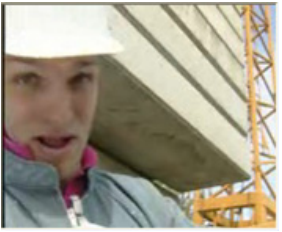

(d) $256 \mathrm{~Kb} / \mathrm{s}, \operatorname{ALG}(\epsilon)$

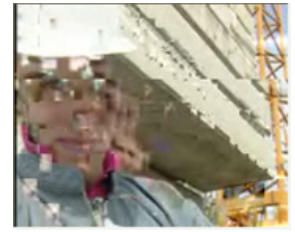

(e) $256 \mathrm{~Kb} / \mathrm{s}, 2-\mathrm{SP}$

Fig. 7. Frame 278 from the reconstructed video.

compare it to the reconstructed video frames under our ALG $(\epsilon)$ and 2-SP for $R=128 \mathrm{~Kb} / \mathrm{s}$ and $R=256 \mathrm{~Kb} / \mathrm{s}$, respectively in Figure 7(b)-(e). The frames under $\operatorname{ALG}(\epsilon)$ have a visual quality very close to the original frame, while the frames under 2-SP are barely recognizable.

\section{RELATED WORK}

In this section, we review some related work that have not been discussed earlier in the paper. Multipath routing has been a topic of active research over the years. For example, various polynomial time algorithms have been proposed to compute $k$-shortest paths [6]. Other important works include, nodeor link-disjoint path routing [13], [16], and braided multiple path routing [11]. However, most of these algorithms do not explicitly consider optimizing performance at the application layer. The problem of path selection for MD video has recently been explored in [3], [18]. In [3], Begen et al. studied the problem of path selection for DD video in the context of overlay networks, where path selection is formulated as an optimization problem that minimizes video distortion. The problem is solved by an exhaustive search over the exponential solution space. In a recent work [18], Wei and Zakhor present a distributed heuristic for finding two maximally disjoint source trees for double description video streaming in ad hoc networks.

\section{CONCLUSIONS}

In this paper, we studied the problem of how to route MD video over multi-hop wireless networks with the objective of optimizing the application layer performance. We formulated this problem into an cross-layer optimization problem with an application performance metric as the objective function and routing and link layer considerations as constraints. We developed a formal branch-and-bound solution procedure and employed a novel relaxation technique called RLT in the solution procedure. We showed that this solution procedure is able to produce a set of routes with an objective value within $(1-\epsilon)$ of the optimal value. Simulation results demonstrated the efficacy of the proposed solution procedure.

\section{REFERENCES}

[1] M. Alasti, K. Sayrafian-Pour, A. Ephremides, and N. Farvardin. Multiple description coding in networks with congestion problem IEEE Trans. Information Theory, 47(3):891-902, March 2001.

[2] J.G. Apostolopoulos, T. Wong, W. Tan, and S. Wee. On multiple description streaming in content delivery networks. In Proc. IEEE INFOCOM, pp. 1736-1745, New York, NY, June 2002.

[3] A.C. Begen, Y. Altunbasak, O. Ergun, and M.H. Ammar. Multi-path selection for multiple description encoded video streaming. EURASIP Signal Processing: Image Commun., 20(1):39-60, Jan. 2005.

[4] J. Chakareski, S. Han, and B. Girod. Layered coding vs. multiple descriptions for video streaming over multiple paths. In Proc. ACM Multimedia, pp. 422-431, Berkeley, CA, Nov. 2003.

[5] T. Clausen and P. Jacquet. Optimized Link State Routing Protocol, Oct. 2003. IETF RFC 3626.

[6] D. Eppstein. Finding the $k$ shortest paths. SIAM J. on Comput., 28(2):652-673, August 1999.

[7] M.R. Garey and D.S. Johnson. Computers and Intractability - A Guide to the Theory of NP-Completeness. New York,NY: W.H. Freeman, 1979.

[8] D.B. Johnson, D.A. Maltz, and Y.-C. Hu. The dynamic source routing protocol for mobile ad hoc networks (DSR), July 2004. IETF Internet Draft, draft-ietf-manet-dsr-10.txt.

[9] S. Mao, Y.T. Hou, X. Cheng, H. D. Sherali, and S.F. Midkiff, Multipath routing for multiple description video in wireless ad hoc networks. In Proc. IEEE INFOCOM, pp.740-750, March 2005, Miami, FL.

[10] S. Mao, S. Lin, S.S. Panwar, Y. Wang, and E. Celebi. Video transport over ad hoc networks: Multistream coding with multipath transport. IEEE J. Select. Areas Commun., 12(10):1721-1737, Dec. 2003.

[11] S. Murthy and J.J. Garcia-Luna-Aceves. Congestion-oriented shortest multipath routing. In Proc. IEEE INFOCOM, pp.1038-1036, San Francisco, CA, May 1996.

[12] G.L. Nemhauser and L.A. Wolsey. Integer and Combinatorial Optimization. John Wiley \& Sons, New York, NY, 1999.

[13] P. Papadimitratos, Z.J. Haas, and E.G. Sirer. Path set selection in mobile ad hoc networks. In Proc. ACM Mobihoc, pp.1-11, Lausanne, Switzerland, June 2002.

[14] C.E. Perkins, E.M. Belding-Royer and S.R. Das, Ad hoc On-Demand Distance Vector (AODV) Routing, IETF RFC 3561, July 2003.

[15] H.D. Sherali and W.P. Adams. A Reformulation-Linearization Technique for Solving Discrete and Continuous Nonconvex Problems. Boston, MA: Kluwer Academic Publisher, 1999.

[16] H.D. Sherali, K. Ozbay, and S. Subramanian. The time-dependent shortest pair of disjoint paths problem: Complexity, models, and algorithms. Networks, 31(4):259-272, Dec. 1998.

[17] Y. Wang, R.R. Reibman, and S. Lin. Multiple description coding for video delivery. Proc. IEEE, 93(1):57-70, Jan. 2005.

[18] W. Wei and A. Zakhor. Multiple tree video multicast over wireless ad hoc networks. to appear in IEEE Trans. Circuits Syst. Video Technol..

[19] X. Zhu, E. Setton, B. Girod. Congestion-distortion optimized video transmission over ad hoc networks. EURASIP Signal Processing: Image Commun., 20(8):773-783, Sept. 2005.

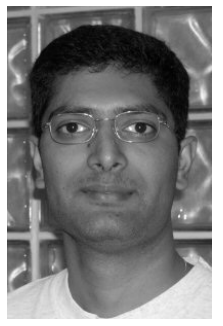

networks.
Sastry Kompella (S'04-M'07) received his B.E. in Electronics and Communication Engineering from Andhra University, India in May 1996, M.S. in Electrical Engineering from Texas Tech University in May 1998, and the Ph.D. degree in Electrical and Computer Engineering from Virginia Tech, Blacksburg, VA, in 2006. Currently, he is a Researcher at U.S. Naval Research Laboratory, Information Technology Division, Washington, DC. His research focuses on complex problems in cross-layer optimizations and multimedia delivery over wireless 


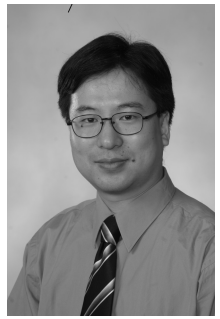

Shiwen Mao (S'99-M'04) received the B.S. and the M.S. degrees from Tsinghua University, Beijing, P.R. China in 1994 and 1997, respectively, both in Electrical Engineering. He received the M.S. degree in System Engineering and the Ph.D. degree in Electrical and Computer Engineering from Polytechnic University, Brooklyn, NY, in 2000 and 2004, respectively. He was a Research Scientist in the Department of Electrical and Computer Engineering, Virginia Tech, Blacksburg, VA during 2004-2006. Currently, he is an Assistant Professor in the Department of Electrical and Computer Engineering at Auburn University, Auburn, AL. Dr. Mao's research interests include cross-layer design and optimization in multi-hop wireless networks, as well as multimedia communications. $\mathrm{He}$ is a co-recipient of the 2004 IEEE Communications Society Leonard G. Abraham Prize in the Field of Communications Systems. He is the co-author of a textbook, TCP/IP Essentials: A Lab-Based Approach (Cambridge University Press, 2004).

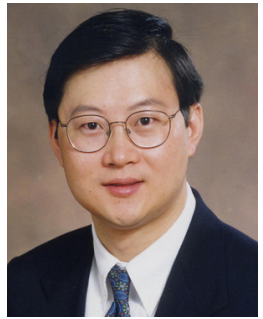

Y. Thomas Hou (S'91-M'98-SM'04) obtained his B.E. degree from the City College of New York in 1991, the M.S. degree from Columbia University in 1993, and the Ph.D. degree from Polytechnic University, Brooklyn, New York, in 1998, all in Electrical Engineering. From 1997 to 2002, Dr. Hou was a Researcher at Fujitsu Laboratories of America, Sunnyvale, CA. Since Fall 2002, he has been an Assistant Professor at Virginia Tech, the Bradley Department of Electrical and Computer Engineering, Blacksburg, VA. His current research interests are radio resource (spectrum) management and networking for software-defined radio wireless networks, optimization and algorithm design for wireless ad hoc and sensor networks, and video communications over dynamic ad hoc networks. Dr. Hou received an ONR Young Investigator Award in 2003 and an NSF CAREER Award in 2004.

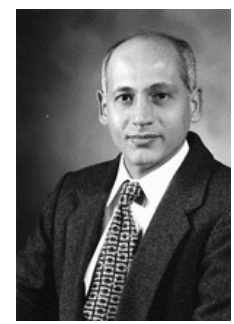

Hanif D. Sherali is W. Thomas Rice Endowed Chaired Professor of Engineering in the Industrial and Systems Engineering Department at Virginia Polytechnic Institute and State University. His area of research interest is in discrete and continuous optimization, with applications to location, transportation, and engineering design problems. He has published over 200 papers in Operations Research journals, has co-authored four books in this area, and serves on the editorial board of eight journals. $\mathrm{He}$ is an IIE Fellow and an INFORMS Fellow, and a member of the U.S. National Academy of Engineering. 